\title{
Research on Present Situation of Market Operation of Gymnastic Dance Club in Neijiang
}

\author{
Weiqiong Zhou ${ }^{1}$ and Zhongfan Zheng ${ }^{1}$ \\ ${ }^{1}$ Institute of Physical Education, Neijiang Normal University, Neijiang, China \\ 374647918@qq.com, 250814033@qq.com
}

Keywords: Gymnastic dance; Club; Market operation; Neijiang

\begin{abstract}
Gymnastic dance is an elegant sport which arouses interest of teenagers, more and more people are involved in it. The paper uses literature method, logical analysis method and field survey method to investigate the present situation of market operation of gymnastic dance club in Neijiang city, analyses the problems existing in the process of marketization, put forward the feasible countermeasures for the related problems, provides theoretical reference for the sustainable development of Neijiang's gymnastic dance club.
\end{abstract}

\section{The Present Development of Gymnastic Dance Club in Neijiang}

The Present Situation of Gymnastic Dance Club in Neijiang. The number and distribution of clubs: Adopt to the investigate, there are various forms of gymnastic dance training, such as weekend interest training and holiday training based on school resourses. The regular clubs has only 16 which located in downtown, there are no clubs have been found in other area. The instrument is the key to club operation. Fully equipped facilities, good environment and safe operation of the club will attract more people. The survey found that the instruments which located gymnastic dance learning and exercising can be basically guaranteed in majority of clubs, but special facilities for gymnastic dance is still deficient. Because of the characteristics of gymnastic dance project, special gymnastic dancing equipment has important effect on the quality and safety of the study and practice. Some clubs are still not required well in the survey.

The Present Situation of Gymnastic Dance Club Coach in Neijiang City. A certain number of sports dance coaches is an important condition for the normal operation of the gymnastic dance club, and the coach team with a better age structure is the guarantee for the sustainable development of the club. In the investigation of several clubs, the total 12 coaches are all part-time coaches,. As part-time instructors can flow, it may bring uncertainty for club teaching and training work. The survey found that the Neijiang city's gymnastic dance club coach's coaching experience and coaching experience should be improved further. Their current salary is not high, gymnastic dance professional coaches are generally paid to salary plus commission class in the form of monthly returns in general in the 4000 - 5000 range, but part-time gymnastic dance coaches are generallypayed above the number of class. Hard working and low income have formed a larger psychological gap; this gap has led the coaches to treat their work negatively, thereby affecting the quality of teaching and training.

Management Status of Gymnastic Dance Clubs in Neijiang City.The current operators engaging in gymnastic dance training is generally engaged in other working sports field before, they can grasp the development of sports fitness industry overall, but because the majority of operators is relatively young, they lack of management tools and management experience to the club operating. Practice has proved that a good sports dance club operators, can consider various aspects of the internal and external factors, such as club construction design, reasonable location and organizational structure, the club staff and facilities equipped with management, market promotion and investment, club sustainable operation. And according to the different problems they can put forward relevant solutions in order to survive and develop in the fierce competition in the fitness market.

The Business Objectives and Strategic Positioning of Gymnastic Dance Club in Neijiang City. With the development of social economy and the improvement of people's living standard, 
people have more leisure time to participate in sports activities; expectations of students' parents promote the development of sports dance industry. Based on the current social form and the characteristics of the sports dance project, the current business objectives of Neijiang gymnastic dance club located in the dance training for youth. At the same time, the clubs are more focused on the events, grading management goal, competition and grading meet youth groups feisty, increase the cultivation of personal specialty skills. The club will work in the game and grading test in a certain period of time. Through this method the club can obtain much business profits. Some clubs also own a student team of gymnastic dance, they perform from city to city to introduce their clubs, create wealth, and practice students' skills as well.

Management Means of Gymnastic Dance Clubs in Neijiang City. Compared with the developed city gymnastic dance club management, Neijiang'sgymnastic dance club management needs to be further improved. First of all, in the information management system, including the members' information, coach's information, employee information, clothing equipment information, the club can't understand the relevant situation in the first time or find out the answer toinformation management. Secondly, the human resources management is more chaotic, subjective and arbitrary characteristics are more prominent. The staff assessment is defected; the salary mode is too fixed. While the developed city's club will examine effects of the staff, adhering to the "customer oriented", "customer is God". They will organize students to evaluate the coach of the club in each stage according to the evaluation results of different classes, implement different bonuses, which can arouse the enthusiasm of the staff in the a large extent.

\section{Influencing Factors of the Development of Gymnastic Dance Clubs in Neijiang}

Poor Hardware Facilities. The character of gymnastic dance determines the needs of venues and better equipment to ensure the safety and effect of the project. Through the investigation we found that the current facilities in Neijiang City gymnastic dance club is not high enough, although the area basically reached the provisions of the Gymnastic Dance Association standards, further investigation shows that the average area of student is still insufficient. A good club environment and cultural atmosphere is the soft power of the development of the club, but the clubs don't pay enough attention to environment constructing, the material culture, institution culture, behavior culture and mental culture construction is deficient.

Gymnastic Dance Teachers Are Weak. In Neijiang, gymnastic dance club coaches' age structure is low, the academic structure is not high, the coach is not long trained. The problem directly affects the development of Neijiang gymnastic dance club. Another survey shows that coach source is more complex, they had engaged in all walks of life, many is lack of specialized training of coaches, some are slack, arrogant, or lack of professionalism and occupation morality in the teaching process, the teaching mode is too single, the training methods are content to follow their own training model ,this directly affects the teaching effect. But in view of the current society of gymnastic dance, instructor training is insufficient, resulting in the shortage of dance coaches, the club cannot pick good coach, they can only maintain the status.

Sports Dance Market, the Main System Are not Perfect. At present, the main body structure of gymnastic dance market in Neijiang city is not perfect, the organizational factors which the body contains is the cornerstone of the main dance market formation,operation and development, , Neijiang city's gymnastic dance clubs have independent operation conditions, autonomic activities, run their own responsibility. But in the standardized operation, because of the market system is not perfect, bad conflicts triggered in the presence of students, price, advertising and other aspects of the competition. At the same time, the current dance training market has not yet formed a club entry and exit mechanism, this lead any individual can rent a shanty area, find a few coaches, operate dance training, there is no standardized evaluation system, we cannot divide the corresponding teaching level according to the level of coaches. 


\section{Countermeasures to Promote the Development of Gymnastic Dance Clubs in Neijiang}

Promote the comprehensive environment of gymnastic dance club. In order to make the club bigger and stronger, we must pay attention to the optimization of the club's hardware resources and the optimization of the club environment. In view of this, in terms of hardware resources, clubs can make full use of some colleges and universities, sign an agreement with the corresponding unit about site using to guarantee gymnastic dance club site. At the same time, as an independent part in the market, club can build their own independent sports venues in permit conditions to prevent the affect by external conditions. In view of the existing problems in the construction of the comprehensive environmental club, the club management needs to pay more attention to the comprehensive improvement of the environment, to establish the concept of "people-oriented" club environment development mechanism, promote the improvement of club culture environment, institutional environment and cultural environment.

Strengthen the Construction of Teaching Staff; Improve the Teaching Level of Coaches. Based on the current situation of Neijiang city gymnastic dance club, club alliance system should be build. Clubs should communicate with the local dance association. Through the cooperation and alliance Club Gymnastic Dance Association, they can build a perfect system of coaches training, regularly organize the theory and practice of learning, and train coach's good occupation morals. They can put forward to "go out, invite in" policy, organize coaches to the developed area for the training, or invite famous coaches to Neijiang to introduce their experience. Through these training and learning method they can make the coaches clearly understand the development trend of the sports dance market, recognize the role and importance of the coaches in club training.

Improve the Club Related System, and Promote the Healthy Development of the Club. In view of the current Neijiang dance club is not perfect, resulting in the club's internal friction increases, the influence of the club development issues, club management should establish and adapt to the development of the club membership system, staff system, equipment system, protect the rights and obligations of employees make sure the students' learning and training process safety and their legitimate rights, so that employees can work in rule-based process, make use of space and equipment to maximize use are reasonable, guarantee the development of gymnastic dance club's improvement, promote sustainable health club development.

Standardize the Standardization of Sports Dance Club Fee Transparency. Because there is no corresponding regulatory mechanism, there are many not according to the notice in the actual operation Bad competition leads to a price war, students of different technology, different personnel have different prices, the main reason for the confusion caused by charges is the assessment fee standard and exercise fee standard is miss, lack of supervision and the corresponding charges standards. The sports dance regulatory authorities need to specified standard fees, charges, training of dance clothing costs charges transparent and standardized, the sports dance training market benign development path on the introduction of fees, and promote the healthy development of the sports dance club training market.

\section{Conclusion}

The paper investigate current situation of Neijiang City Sports Gymnastic Club: the club number, club size, number and distribution of the current situation of coaches, dance club business status, business strategy, management means, management efficiency and other aspects of the investigation and analysis, shows that the restrict factors in the development of Neijiang City Dance Club factors are: the bad quality of the team, bad quality of club coaches, weak hardware facilities gymnastic dance market system is not perfect. The paper puts forward some countermeasures to promote the development of Neijiang City Gymnastic Dance Club: to promote the comprehensive environment, improve and strengthen the construction of teaching staff and improve the teaching level of the coaches, improve the relevant system to promote the healthy development of the club, the club standard fees make sports dance club charges transparent strategy. 


\section{Acknowledgements}

This work is supported by Scientific research project of Neijiang Normal University (Number: 14SB04)

\section{References}

[1] Chen Linong.Research on the present situation and Countermeasures of sports dance in China [J].Journal of Guangzhou Sports University, 2001, (01).

[2] Jiang Xiaoping. Review and Prospect of research on sports dance in China [J]. Journal of Physical Education Institute of Shanxi University, 2005, (02).

[3] Liu Gengliang, Duan Liang. Analysis of Sports Dance Project in Shandong Province [J].Journal of Shandong Institute of Physical Education and Sports, 2011, (01).

[4] Wang Jun, Li Xiaoyan. Investigation and Analysis on the current situation of youth sports dance in Yichang [J]. Journal of Xi'an Physical Education University, 2003, (010).

[5] Zhao Li. Investigation and countermeasure research on the present situation of sports dance in China [D] Master's degree thesis of Beijing Sport University, 2003. 